\title{
Repentinas como estrategia didáctica para el desarrollo de la creatividad en los procesos iniciales de la enseñanza de arquitectura
} Sudden workshops as a didactic strategy for the development of creativity in the initial processes of teaching architecture

Enmanuel Salazar-Ceciliano ${ }^{1}$, Jeannette Alvarado-Retana ${ }^{2}$, Dominique Chang-Albizurez ${ }^{3}$

Salazar-Ceciliano, E; Alvarado-Retana, J; Chang-Albizurez, D. Repentinas como estrategia didáctica para el desarrollo de la creatividad en los procesos iniciales de la enseñanza de arquitectura. Tecnología en Marcha. Edición especial.

Escuela de Arquitectura y Urbanismo, Diciembre 2020.

Pág 71-78.

doi) https://doi.org/10.18845/tm.v33i8.5510 


\title{
Palabras clave
}

Educación; diseño arquitectónico; creatividad; experimento educacional; proceso de aprendizaje.

\section{Resumen}

La creatividad es una de las más reconocidas habilidades de los arquitectos, y, por tanto, su desarrollo figura como uno de los principales retos durante el proceso de aprendizaje. Teorías como las que comparan el proceso creativo con una caja negra han sido apoyadas durante décadas. En esta, el diseñador es capaz de obtener resultados satisfactorios, pero no es capaz de fundamentar los insumos que lo llevaron al objeto final o replicar el procedimiento en otro proyecto. Por el contrario, también han surgido métodos que definen la creatividad como una caja transparente, donde existe una rigurosa investigación previa que le permite al diseñador llenarse de recursos y variables que dan partida a su proceso proyectual.

Partiendo de este enfoque, es común ver a los estudiantes, principalmente a los de primeros años, agobiados ante el papel en blanco y diversos factores que aún no pueden interpretar o relacionar. Por tanto, este documento plantea una metodología que implementa las repentinas, o ejercicios rápidos en clase, como una herramienta para que los estudiantes tomen decisiones de diseño sustentadas en su investigación previa y den partida a su proceso creativo. Lo planteado se basa en la experiencia llevada a cabo en el Laboratorio de Arquitectura II de la Escuela de Arquitectura y Urbanismo del Tecnológico de Costa Rica durante el segundo semestre del 2019.

\section{Keywords}

Education; architecture design; creativity; educational experiments; learning processes.

\begin{abstract}
Creativity is one of the most recognized skills of architects, therefore, its development is one of the main challenges during the learning process. Theories like those comparing the creative process to a black box have been supported for decades. In this, the designer obtains satisfactory results but is not able to base the inputs that led him to the final object or to replicate the steps in another project. On the contrary, another method that defines creativity as a glass box has emerged, where there is rigorous prior research that allows the designer to fill himself with resources and variables to start his projectual process.

Starting from this approach, it is common to see students, mainly those in the first year, overwhelmed by the blank paper and various factors that they still cannot interpret or relate. Therefore, this document presents a methodology that implements sudden workshops, or quick exercises in class, as a tool for students to make design decisions supported on their previous research and start their creative process. The proposal is based on the experience carried out in the Laboratory of Architecture II of the Architecture and Urbanism School of the Costa Rica Institute of Technology during the second semester of 2019.
\end{abstract}

\section{Introducción}

La arquitectura se ha caracterizado por sus resultados innovadores y bellos, que además de brindar las condiciones necesarias para que los seres humanos puedan desarrollar sus actividades cotidianas, también asombra y acoge a todos a aquellos que la recorren y se refugian en ella. La arquitectura ha estado muy ligada a la ciencia y al arte, mezclando procesos científicos, para poder emplear nuevas técnicas, y procesos subjetivos, para agradar a los usuarios. 
El diseño arquitectónico está alimentado en gran medida por la creatividad del diseñador, la cual resulta ser un fenómeno dinámico y libre [1] que ha intentado ser definido por múltiples autores a lo largo de la historia. Mark Runco y Garrett Jaeger atribuyen al trabajo de Morris Stein publicado en 1953 la definición más común de creatividad, la cual se centra en dos aspectos fundamentales: la originalidad y la eficiencia [2].

Se entiende por originales a aquellas ideas novedosas o raras, es decir, de poca frecuencia en comparación con otras [3], mientras que la efectividad es vista como el hecho de alcanzar metas o servir para resolver un propósito específico, si se analiza desde un modelo de cumplimiento de objetivos [4]. Ambas definiciones son fáciles de comprobar a la hora de evaluar un producto, pero cómo entrenar a un diseñador para que pueda impregnar de estas características a cada una de sus ideas ha sido un reto que la academia, todavía hoy, sigue asumiendo.

El proceso creativo puede ser abordado desde un punto de vista subjetivo y otro objetivo, es decir, totalmente racional. Desde el enfoque subjetivo se clasifica al diseñador como una caja negra, la cual es capaz de entender un problema y brindar una solución, pero donde el proceso para producirla es desconocido [5]. El diseñador está mayormente vinculado al pensamiento heurístico o hermenéutico, y además de necesitar de mucho trabajo para arrojar un resultado satisfactorio, hace uso de conocimientos previos que almacena en su memoria [6].

Por el contrario, la visión objetiva propone al diseñador como una caja transparente, donde el uso del pensamiento algorítmico permite trazar el proceso creativo, creando una secuencia de recopilación de información, análisis, síntesis, evaluación y producción [6]. Este modelo también permite familiarizar al estudiante con procesos de investigación, que le ayuden a encontrar todos los insumos que necesite para emprender su proceso proyectual, situación que deberá realizar por sí mismo cuando se encuentre ejerciendo como profesional.

Los arquitectos, en buena parte, se sienten más identificados con las teorías de la creatividad de la caja negra y el arte, lo que ha llevado a concebir la profesión como un campo de pocas regulaciones y amplia libertad por parte del diseñador [7]. Esta situación suele generar confusión en los estudiantes, principalmente en los primeros años, ya que vienen de un sistema educativo que les da indicaciones claras de lo que tienen que hacer y cómo deben realizarlo.

Es por esa razón que, con la visión de que el estudiante debe buscar elementos físicos y perceptuales para concebir una idea espacial [8] que sea novedosa y eficiente, en el Laboratorio de Arquitectura II, de la Escuela de Arquitectura y Urbanismo del Instituto Tecnológico de Costa Rica, se estableció una metodología basada en la realización de repentinas o trabajos rápidos de clase, que al estar fundamentados en una investigación previa, le dé al estudiante la confianza de tomar decisiones de diseño, así como le permitan llegar a soluciones propias del sitio donde trabaja y más pertinentes a las necesidades de su usuario.

\section{Materiales y métodos (metodología)}

Como técnicas metodológicas principales se utilizaron el "análisis de protocolo de diseño" (design protocol analysis method -DPA) [9] [10], que incluye el análisis de gráficos, discursos y diagramas; los argumentos y procesos de fundamentación- explicación; las operaciones de exploración espacial para llegar a la formalización y materialización de la solución proyectual, los procesos de inferencia lógica: razonamiento práctico y teórico; y la expresión de la solución proyectual (láminas y maquetas) [11]. Además, se empleó la observación del proceso proyectual de los estudiantes en el aula.

Los participantes fueron los estudiantes y los profesores de los 2 grupos del curso de Laboratorio de Arquitectura I de la carrera de Arquitectura de Escuela de Arquitectura y Urbanismo del 
Instituto Tecnológico de Costa Rica, durante el segundo semestre del 2019. En total participaron 48 estudiantes y 3 docentes.

Para que todos los estudiantes estuvieran en igualdad de condiciones y la evaluación fuera objetiva y homogénea, los proyectos realizados en el curso tenían: un sitio, un programa, una necesidad, y una tipología formal o estructural que guiara el proceso proyectual. En total se realizaron 4 ejercicios durante el semestre, en donde se trabajaron las siguientes tipologías: planos seriados, tenso estructuras y voladizos o cantiléver. Solamente se efectuaron 3 repentinas.

Cada proyecto se abordó en 3 etapas. La primera correspondía a la fase de investigación y análisis de sitio, en donde tanto estudiantes como profesores, visitaban el terreno en donde se desarrollaría la propuesta espacial para realizar un levantamiento de datos, según los criterios de análisis establecidos por María Concepción Chong. América Carmona y Marco Antonio Pérez, los cuales clasifican los elementos de un lugar en dos sistemas: el físico-biótico y el sociocultural [9]. Luego de realizado el levantamiento, se precede a un análisis y síntesis de la información, el cual determina los requerimientos del proyecto y los principios de orden que condicionarían el proceso proyectual.

La segunda fase consistió en la conceptualización de la propuesta, en donde el estudiante empieza a manipular los requerimientos de diseño y principios de composición para establecer su primera propuesta espacial. Es justamente en esta etapa donde se implementaron las repentinas, o ejercicios rápidos en clase.

El día en que se realizaba la repentina, el estudiante debía llegar a clase con su bitácora y el análisis realizado en la etapa anterior. No es sino hasta este día que se revela la tipología formal y estructural con la que se trabajará el proyecto, por lo que durante la primera hora de clase se hace una mediación de contenidos sobre las implicaciones estructurales y de diseño que tiene la tipología a abordar. El objetivo de no revelar esta información durante el análisis de sitio es evitar que los estudiantes prefiguren una propuesta arquitectónica sin haber realizado una investigación previa. Luego de esta charla, los estudiantes tienen cerca de dos horas para concebir un primer acercamiento de su partido arquitectónico.

Los materiales para utilizar durante esta etapa dependen de la tipología arquitectónica a desarrollar. Por ejemplo, mientras que para los proyectos de planos seriados y voladizos se emplearon cartones y madera de balsa, para el ejercicio de tenso estructuras se emplearon telas y tensores.

La evaluación de la repentina examina tres aspectos fundamentales: el uso del material solicitado por los profesores, ya que sin la existencia de este requisito no se podría realizar la dinámica de clase; el compromiso con la actividad, es decir, que el estudiante muestre interés durante el tiempo designado y que no distraiga a sus demás compañeros; y la coherencia entre el contexto con el encargo, donde el estudiante debía demostrar la utilización de los resultados de la investigación previa en la creación de su propuesta espacial. Como se puede observar, los criterios de evaluación no buscan emitir criterio subjetivo sobre si el resultado es bueno o malo, sino motivar a los jóvenes a participar activamente de la dinámica y emplear todos los recursos que han generado durante el proceso. Una vez cumplido el tiempo asignado, los estudiantes se retiran de la clase para que los profesores puedan evaluar rápidamente, y cuando regresan se da paso a un espacio de discusión en donde todos puedan presentar sus ideas.

Durante el último ejercicio no se realizó la repentina, para probar si los estudiantes ya habían interiorizado el procedimiento y eran capaces de realizar por su cuenta los pasos establecidos sin ser una obligación del curso. 
En todos los ejercicios se realizó la repentina después de la segunda semana de iniciado el proyecto, y una vez finalizado el análisis del sitio. En cada ejercicio se aumentó la cantidad de variables a considerar, tanto en el análisis como en la propuesta espacial.

La tercera etapa del proyecto consistió en la depuración del proyecto arquitectónico, así como la realización de planos y modelos de presentación, los cuales son exhibidos públicamente 2 semanas después siguiendo el formato establecido.

Para evaluar los resultados de la aplicación de la repentina como estrategia didáctica se toman como parámetro tres factores:

Nivel de ansiedad de los estudiantes al inicio de la dinámica y grado de satisfacción con respecto a los resultados. Al finalizar cada proyecto se aplicó un breve cuestionario de Google Forms para identificar la percepción de los jóvenes con respecto a las estrategias empleadas.

La evolución de los resultados de la repentina con respecto a la entrega final del proyecto. Los profesores dieron seguimiento a los resultados de la repentina de cada estudiante durante el resto del proceso de diseño, y registran cuales proyectos finales muestran una clara relación con el modelo de la repentina.

Al terminar el semestre, se analizaron las evaluaciones finales de todos los ejercicios para identificar la eficacia de la metodología planteada.

\section{Resultados}

Desde el punto de vista didáctico, se plantearon ejercicios que se centran en la actividad de diseño como tema-objeto de aprendizaje desde una actitud reflexiva y crítica, de manera que los estudiantes sean conscientes de sus propios procesos de respuesta y tomen decisiones que intervienen en la solución ejercicios que no tienen una única respuesta.

En el primer año el estudiante inicia modelando una situación problemática de intervención, que depende en gran medida de la formulación explícita de un problema de diseño que los profesores plantean a nivel de encargo, para que los estudiantes creen modelos interpretativos de la demanda o necesidad del usuario planteado. De alguna manera se espera que el estudiante cree nuevas categorías conceptuales que "encarnen" simbólicamente los objetos arquitectónicos [11].

El primer ejercicio planteado fue un proyecto sencillo, con tres funciones básicas, ubicado en un contexto de poca complejidad, y teniendo como condicionante particular el uso de planos seriados; el ejercicio duró tres semanas. La repentina como estrategia didáctica se aplicó para generar uno de los planos que conformaría el objeto arquitectónico solicitado. Se observó que al ser la primera vez que los estudiantes eran enfrentados a esta estrategia didáctica, antes de iniciar la actividad, alrededor del 54\% de los jóvenes expreso sentir ansiedad en cuanto no sentirse capaces de obtener resultados en un lapso tan corto. Enfrentarse a la complejidad, la indeterminación y la incertidumbre son parte de lo implícito en lo que Guevara denomina intención [7]. Una vez finalizada la repentina el 23\% de los estudiantes lograron una primera propuesta espacial que evolucionó y quedo reflejada en la propuesta final. El $77 \%$ de los jóvenes expresó que, si bien el resultado obtenido no era satisfactorio, en términos de haber logrado un plano seriado que pudieran utilizar en la propuesta formal final, el ejercicio les había ayudado a generar una respuesta concreta en corto tiempo, a partir de la cual era posible continuar explorando el resultado para llegar a una propuesta que expresara de mejor manera su intención formal.

El segundo ejercicio tuvo una duración de cuatro semanas y forzó a la exploración volumétrica en un proyecto igualmente sencillo, con una función básica, ubicado en un contexto de clima 
caliente ligeramente más complejo que el primero, y tuvo como condicionante particular el uso de tenso estructuras o velarias. La repentina como estrategia didáctica en este ejercicio fue aún más enriquecedor para los estudiantes, ya que en este nivel su expresión gráfica aún es limitada, y permitirles explorar en un modelo desde el inicio facilitó la obtención de propuestas formales de manera más ágil. Teniendo la experiencia del ejercicio anterior, el nivel de ansiedad al arranque de la actividad disminuyó en un 16.5\%, es decir, solo el $37.5 \%$ sintió ansiedad antes de iniciar la repentina. Una vez finalizada la dinámica, el $42 \%$ de los estudiantes logró una primera propuesta espacial que evolucionó y quedó reflejada en la propuesta final presentada. El 83\% de los estudiantes expreso sentirse satisfecho con el resultado obtenido, en el sentido que les facilitaba continuar con la exploración espacial, partiendo de una experimentación que, de haber realizado por su cuenta sin recibir la realimentación de los profesores y compañeros, no hubieran podido alcanzar.
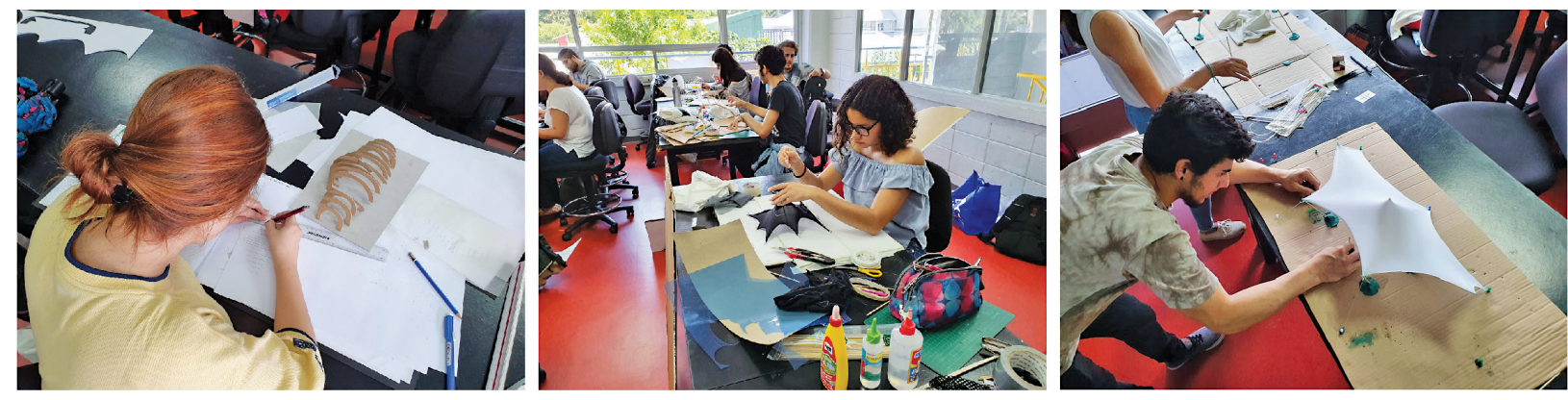

Figura 1. Estudiantes trabajando durante las repentinas 1 y 2. Fotografías de autoría propia.

El tercer ejercicio fue más complejo en cuanto a función, por lo que incluyó dos etapas: la primera consistía en diseñar un módulo habitacional sencillo y la segunda en proyectar a nivel de sitio el conjunto de los módulos generados en la primera etapa más el diseño de un recinto para actividades comunes. El ejercicio tuvo una duración de cinco semanas y como tipología arquitectónica establecida se debía diseñar bajo los principios del cantiléver o voladizos, teniendo por contexto un terreno con pendiente. La repentina se utilizó únicamente en la primera etapa.

El nivel de ansiedad bajo a un 18\% antes de iniciar la repentina. Una vez finalizada, el 52\% de los estudiantes logró una propuesta espacial que evolucionó y quedó reflejada en la propuesta final. El $86 \%$ de los estudiantes expresó sentirse satisfecho con la realización de la actividad, aunque no lograron incorporar todas las variables requeridas en esa primera propuesta espacial.

Como se puede observar en la figura 2, el nivel de satisfacción de los estudiantes fue aumentando con cada repentina, mientras disminuía su nivel de preocupación ante el ejercicio. Con la tercera dinámica, cerca de la mitad de los estudiantes lograron un primer acercamiento espacial satisfactorio que siguió siendo trabajado hasta la entrega final, mientras que la otra mitad tuvo que replantear sus ideas.

El cuarto ejercicio corresponde a la etapa dos del proyecto antes mencionado, en donde los estudiantes debían generar una propuesta de sitio más un espacio de amenidades para los habitantes del complejo que estaban diseñando. En esta ocasión no se realizó la repentina como dinámica de clase, sino que se fueron atendiendo de forma más personalizada a los estudiantes para guiar el avance. Al finalizar, al menos un 38\% de los jóvenes manifestaron haber echado de menos la repentina durante su proceso creativo, ya que no contaban todavía con la seguridad suficiente como para realizar la dinámica de manera independiente. 
Un 40\% manifestó haber seguido por su cuenta un proceso similar al realizado durante los ejercicios anteriores, obteniendo resultados satisfactorios, sin embargo, manifestaron extrañar el intercambio de ideas entre compañeros y profesores que se efectuaba durante la repentina.

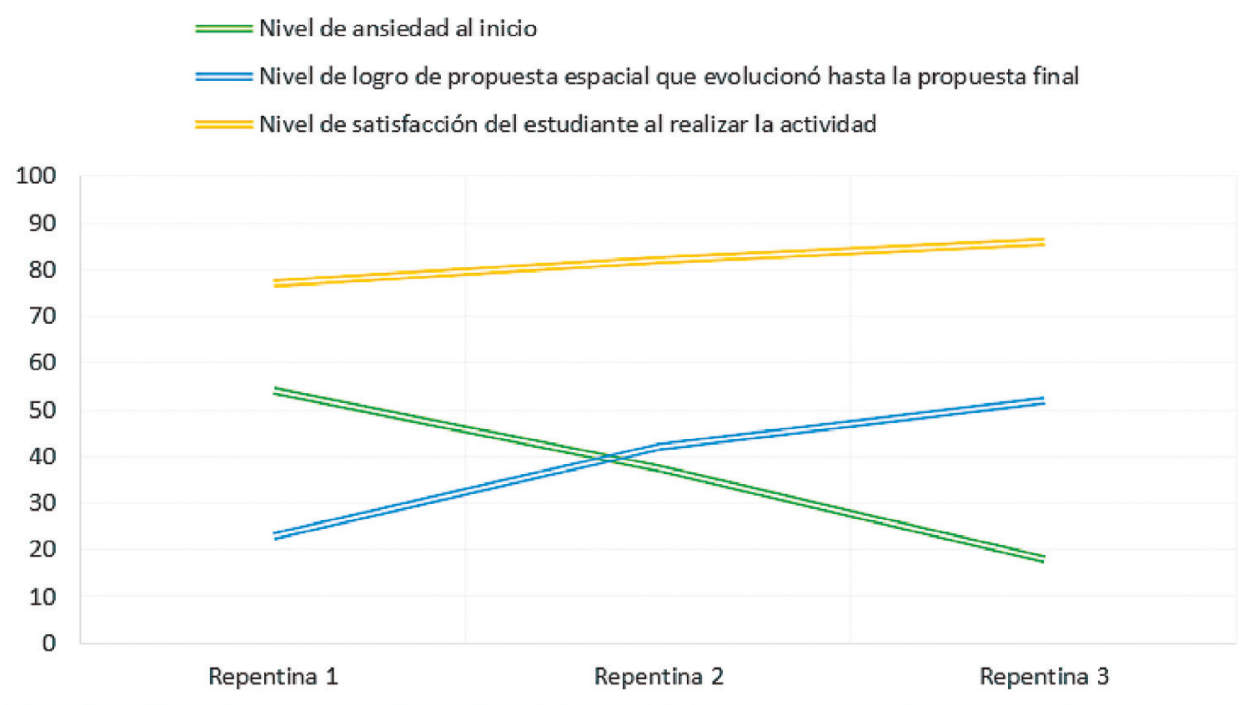

Figura 2 Niveles de ansiedad y logros de estudiantes durante cada repentina.

\section{Conclusiones}

La metodología propuesta promueve la condición propositiva y creativa de la arquitectura en su contexto de enseñanza, enmarcada en la premisa planteada, intereses y trayectoria de los docentes de los primeros niveles de la disciplina y actividades propias de la Escuela de Arquitectura y Urbanismo del Instituto Tecnológico de Costa Rica.

De modo significativo la metodología aplicada genera nuevas posibilidades de exploración de la forma propuesta apoyada en el argumento del contexto, el programa o situaciones particulares del lugar, el usuario, la cultura, la construcción y su materialidad como factores que jerarquizan y ordenan su desarrollo.

En el método de repentinas o trabajos cortos se visualizan dos aristas, una referente al encargo que se propone y argumenta y, otra, a la solicitud que se hace al estudiante para que ejercite su capacidad de ver y ejecutar en condiciones propias de la arquitectura, por medio de ejercicios básicos, elementales que van ejecutando, profundizando y complejizando a través del curso lectivo.

Las repentinas se plantearon bajo una estrategia didáctica que busca ayudar a los estudiantes de los primeros niveles, a partir de la experimentación de una nueva forma de abordaje del objeto-tema y una exploración reflexiva y creativa para dar una primera propuesta rápida, apropiada al sitio, acorde con los lineamientos dados y las particularidades de las tipologías arquitectónicas.

Los estudiantes lograron comprender el proceso desde una perspectiva amplia, explorativa, que contrasta con una visión tradicional y limitada del oficio del diseño, y la construcción del pensamiento creativo que surge de la unión del pensamiento asociativo, que ve en los 
errores una oportunidad y que tensiona las reglas, y el pensamiento exacto que comprueba y contrapone el pensamiento asociativo coherente con la conceptualización de caja transparente y caja negra.

De conformidad con lo anterior el grupo de docentes espera que el desempeño académico de estos estudiantes sea superior a los formados con esquemas de especulaciones de la forma desde una visión reducida del estudiante o docente, sin sustento teórico y conceptual.

Los estudiantes demostraron un mayor interés y capacidad de asumir los trabajos evidenciando una disposición superior para el trabajo individual y grupal. Las dinámicas entre docentes y los estudiantes permitieron el intercambio académico ante la diversidad de criterios al enfrentar una misma problemática que derivan en múltiples alternativas de solución según la complejidad arquitectónica solicitada. Lo anterior demuestra que es importante propiciar niveles de exigencia adecuados que estimulen el crecimiento, toma de decisiones, disciplina, y responsabilidad de los estudiantes.

El recurso pedagógico implementado permitió la evaluación de la capacidad creativa para enfrentar y solucionar un problema aplicando conceptos teóricos y los lineamientos dados en cada ejercicio. La evaluación aplicada es fundamental en el momento de aprendizaje y como retroalimentación del estudiante y los docentes, a través, de un registro personalizado de los aportes y avances y un panorama general de los logros del grupo y el curso. Los profesores deberían plantear la evaluación para, además de medir el nivel alcanzado por los estudiantes durante el ejercicio, identificar la eficacia de las estrategias didácticas en los estudiantes, y así estimular al máximo su proceso creativo.

\section{Referencias}

[1] G. E. Corazza, «Potential Originality and Effectiveness: The Dynamic Definition of Creativity,» Creativity Research Journal, vol. 28, n 3, pp. 258-267, 2016.

[2] M. A. Runco y G. J. Jaeger, «The Standard Definitio of Creativity., Creativity Research Journal, vol. 24, n 1 , pp. 92-96, 2012.

[3] N. Mayseless, A. Eran y S. G. Shamay-Tsoory, «Generating original ideas: The neural underpinning of originality,» Neurolmage, vol. 116, pp. 232-239, 2015.

[4] K. Cameron, «Organizational Effectiveness,» Wiley Encyclopedia of Management, vol. 11, pp. 1-4, 2015.

[5] K. E. Jennings, D. K. Simonton y S. E. Palmer, «Understanding Exploratory Creativity in a Visual Domain,» de Proceedings of the 8th ACM conference on Creativity and cognition, 2011.

[6] N. E. NACIF, «Métodos de Diseño,» Universidad Nacional de San Juán, San Juan, 2015.

[7] O. E. Guevara Alvarez, «Análisis del proceso de enseñanza aprendizaje de la Disciplina Proyecto Arquitectónico, en la carrera de Arquitectura, en el contexto del aula.,» Universidad Autónoma de Barcelona, Bellaterra, 2013.

[8] L. M. Franco Cárdenas, I. R. Gutiérrez Ruiz y M. Navarro Velázquez, «La creatividad en la formacion del arquitecto: procesos, herramientas y estrategias,» de La expresión y la representación como lenguaje del diseño, Guadalajara, Universidad de Guadalajara, pp. 189-213.

[9] P. Galle, «Design rationalization and the logic of design: a case study,» Design Studies, vol. 17, n 3, pp. 253275, 1996.

[10] P. Galle y L. B. Kovács, «Replication protocol analysis: a method for the study of real-world design thinking,» Design Studies, vol. 17, n² 2, pp. 181-200, 1996.

[11] C. E. Burgos, «La naturaleza cognitiva del proyecto y la crisis en la concepción heredada en la enseñanza de la arquitectura,» Arquitecturas del sur, vol. 33, n 48, pp. 44-55, 2015.

[12] M. C. Chong Garduño, A. Carmona Olivares y M. A. Pérez Hernández, «El análisis de sitio y su entorno en el desarrollo de proyectos arquitectónicos y urbanos,» 2012. 